\title{
Organisierte Inkompetenz bei Wirtschaftlichkeitsprüfung der SIT
}

D ie Verordnung von Therapielösungen zur spezifischen Immuntherapie im vertragsärztlichen Bereich hat sich wie alle Therapieformen an den Vorgaben des Arzneimittelgesetzes zu orientieren. Die Vorgaben des Wirtschaftlichkeitsgebots nach $\$ 12$ SGB V und die Richtlinien des Bundesausschusses der Ärzte und Krankenkassen über die Verordnung von Arzneimitteln in der vertragsärztlichen Versorgung (Arzneimittel-Richtlinien/AMR) reglementieren die Therapie im Bereich der GKV. Medizinisch-fachlich fasst die durch die allergologischen Fachgesellschaften gemeinsam erstellte S2-Leitlinie „Die spezifische Immuntherapie..." (Allergo J 2006; 15: 56-74) das derzeitige Wissen über diese Therapie zusammen.

Soweit alles klar und eindeutig - sollte man meinen! Allerdings wurden in jüngster Zeit von einigen Prüfgremien Aussagen der Leitlinie in einer Art interpretiert, die in erschreckendem Ausmaß eine fehlende Fachkompetenz dieser Gremien verdeutlichen. So wird in der Leitlinie der Um-

„Die allergologischen Fachgesellschaften werden weiterhin alles Erdenkliche tun, um Schaden von den Kollegen abzuwenden." stand angeführt, dass SIT-Präparate nach gültigem Gesetzesstandard entweder als Fertigarzneimittel über das Paul-

Ehrlich-Institut zugelassen sein oder aber gemäß $\$ 21$ Absatz 2, 1 b BAMG als Individualrezepturen auf dem deutschen Markt vertrieben werden können.

Aus diesen Ausführungen folgerten die Prüfausschüsse folgenschwer, dass die Verwendung von nicht zugelassenen Präparaten unwirtschaftlich und der medizinische Nutzen nicht erwiesen sei. Diese Interpretation der Aussage der Leitlinie ist willkürlich und sachlich falsch! Dennoch wurden auf dieser Grundlage Regressforderungen in schwindelerregender Höhe ausgesprochen. Grundsätzlich gilt, dass zwischen einem als Fertigarzneimittel zugelassenen Immuntherapiepräparat und Therapeutikum, das im Rahmen einer Individualrezeptur hergestellt wurde, weder automatisch Qualitätsunterschiede bestehen, noch dass ein zugelassenes Präparat preislich günstiger ist.
Die allergologischen Fachgesellschaften haben sich daher mit Nachdruck für die betroffenen Kollegen eingesetzt und werden weiterhin alles Erdenkliche tun, um Schaden von allen Kollegen abzuwenden, die mit sachlich begründeten Therapien ihre Patienten gut versorgen. Hierzu wird voraussichtlich noch in diesem Jahr eine ClearingStelle beim Ärzteverband Deutscher Allergologen eingerichtet.

Da es sich bei Präparaten zur SIT um verhältnismässig teure Substanzen handelt, werden gerade Arztgruppen mit geringen Arzneimittel-Richtgrössen wie $\mathrm{HNO}$ - und Hautärzte bei einem hohen Anteil allergologischer Patienten von den derzeitigen Regelungen benachteiligt.

Glücklicherweise enthält dieses Heft auch viele erfreuliche Aspekte allergologischen Wirkens, die es trotz der politischen Seitenhiebe ja weiterhin gibt!

Wir wünschen Ihnen viel Spass bei der Lektüre, Ihre
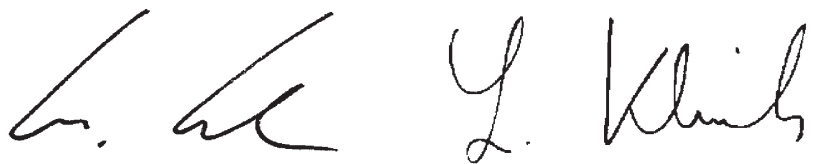

Prof. Dr. Wolfgang Czech
Prof. Dr. Ludger Klimek 